\title{
Vascular endothelial dysfunction resulting from $L$-arginine deficiency in a patient with lysinuric protein intolerance
}

\author{
Yoshihiro Kamada, Hiroyuki Nagaretani, Shinji Tamura, Tohru Ohama, \\ Takao Maruyama, Hisatoyo Hiraoka, Shizuya Yamashita, Akira Yamada, Shinichi Kiso, \\ Yoshiaki Inui, Nobuyuki Ito, Yoshiro Kayanoki, Sumio Kawata, and Yuji Matsuzawa
}

Department of Internal Medicine and Molecular Science, Graduate School of Medicine, Osaka University, Osaka, Japan

Address correspondence to: Shinji Tamura, Department of Internal Medicine and Molecular Science, Graduate School of Medicine, Osaka University, B5, 2-2, Yamada-oka, Suita, Osaka 565-0871, Japan. Phone: 81-6-6879-3732; Fax: 81-6-6879-3739; E-mail: tamuras@imed2.med.osaka-u.ac.jp.

Yoshihiro Kamada and Hiroyuki Nagaretani contributed equally to this work.

Received for publication September 6, 2000, and accepted in revised form July 20, 2001.

\begin{abstract}
Although L-arginine is the only substrate for nitric oxide (NO) production, no studies have yet been reported on the effect of an L-arginine deficiency on vascular function in humans. Lysinuric protein intolerance (LPI) is a rare autosomal recessive defect of dibasic amino acid transport caused by mutations in the SLC7A7 gene, resulting in an L-arginine deficiency. Vascular endothelial function was examined in an LPI patient who was shown to be a compound heterozygote for two mutations in the gene (5.3-kbp Alu-mediated deletion, IVS3 $+1 \mathrm{G} \rightarrow \mathrm{A}$ ). The lumen diameter of the brachial artery was measured in this patient and in healthy controls at rest, during reactive hyperemia (endotheliumdependent vasodilation [EDV]), and after sublingual nitroglycerin administration (endotheliumindependent vasodilation [EIV]) using ultrasonography. Both EDV and $\mathrm{NO}_{\mathrm{x}}$ concentrations were markedly reduced in the patient compared with those for the controls. They became normal after an L-arginine infusion. EIV was not significantly different between the patient and controls. Positron emission tomography of the heart and a treadmill test revealed ischemic changes in the patient, which were improved by the L-arginine infusion. Thus, in the LPI patient, L-arginine deficiency caused vascular endothelial dysfunction via a decrease in NO production.
\end{abstract}

J. Clin. Invest. 108:717-724 (2001). DOI:10.1172/JCI200111260.

\section{Introduction}

In 1980, Furchgott and Zawadzki demonstrated that the relaxation of vascular smooth muscle cells in response to acetylcholine is dependent on the anatomical integrity of the endothelium. They referred to the factor that is responsible for this intercellular relationship as endothelium-derived relaxing factor (EDRF; ref. 1). EDRF was identified 7 years later as the free radical gas nitric oxide (NO; refs. 2, 3). NO is produced from L-arginine enzymatically via the action of nitric oxide synthase (NOS; ref. 3). NO exerts a variety of physiological effects that include vasodilation (3), a decrease in gastrointestinal motility (4), macrophage-induced cytotoxicity (5), and the inhibition of platelet aggregation (6). NO is also important in terms of vascular endothelial function (7-9). The regulation of the endothelium can be altered by cardiovascular risk factors or disorders, such as hypertension (10), hypercholesterolemia (11-13), chronic smoking (14), diabetes mellitus (15), or coronary artery disease $(16,17)$. L-Arginine improves endothelial dysfunction in these disorders (10-13, 15-17). Because $\mathrm{L}$-arginine is the only physiological nitrogen donor for the NOS-catalyzed reaction (18), the availability of this essential substrate would be expected to determine the cellular rate of NO synthesis. An L-arginine deficiency could, therefore, lead to a decrease in NO production. However, because L-arginine is, nutritionally, a nonessential amino acid for adult mammals including humans (19), to our knowledge no investigations have been reported relative to the effect of a deficiency of this substrate on NO synthesis in humans except for our previous report (20). The effects of NO deficiency on vascular endothelial function using chronic experimental NO inhibition by L-arginine analogues have been reported (21). However, no information is presently available on vascular function for an L-arginine deficiency in vivo.

Lysinuric protein intolerance (LPI; online Mendelian inheritance in man 222700) is an autosomal recessive disease caused by defective dibasic amino acid (DAA; L-arginine, L-lysine, L-ornithine) transport at the basolateral membrane of the epithelial cell (22). LPI is characterized by symptoms that include failure to thrive, hepatosplenomegaly, muscle hypotonia, sparse hair, osteoporosis, and life-threatening pulmonary involvement (alveolar proteinosis). Mutations in the solute carrier family 7A member 7 (SLC7A7) gene are the cause of LPI $(23,24)$. This mutation results in a deficiency of DAA, and as a result, LPI patients are deficient in L-argi- 
nine. Thus, patients with LPI may have an impaired function of the vascular endothelium.

In the current study, we describe, for the first time to our knowledge, vascular endothelial dysfunction in an LPI patient with an L-arginine deficiency.

\section{Methods}

Subjects. A 37-year-old Japanese man presented typical clinical findings of LPI that included increased urinary excretion and a low plasma concentration of $\mathrm{L}$-arginine, L-lysine, and L-ornithine, as has been reported previously (20). His fasting plasma glucose and serum total cholesterol were normal (Table 1), but he frequently complained of chest pains.

We examined the patient, his mother, and one normal control by means of a mutation analysis. The patient's father died 1 year ago, and it was not possible to investigate his genetic make-up.

Vascular endothelial function was examined before and after an L-arginine infusion in the patient and in ten healthy men between the ages of 30 and 42 years (mean age: $35.3 \pm 4.2$ years) as normal controls (control A). Endothelium independent vasodilation after nitroglycerin administration was also measured in the patient and in ten healthy men between the ages of 25 and 44 years (mean age: $36.3 \pm 4.7$ years) as normal controls (control B). The patient had not received L-arginine or nitroglycerin for at least 2 weeks prior to this study. The patient and normal controls were all nonsmokers and were receiving no medication. No significant differences were evident in the baseline values of heart rate and blood pressure between the patient and the controls (Table 1).

All subjects in this study provided written informed consent.

RT-PCR, genomic DNA amplification, sequencing, and mutation analysis. DNA and total RNA were extracted from peripheral blood leukocytes from whole blood using the QIAGEN Blood \& Cell Culture DNA Midi kit and QIAamp RNA Blood Minikit (both, QIAGEN $\mathrm{GmbH}$, Hilden, Germany) following the manufacturer's recommended protocols.

The cDNA sequence of the SLC7A7 gene was obtained from GenBank AJ130718. Oligonucleotide primers based on the cDNA sequence were initially used. Additional oligonucleotide primers were prepared on the basis of the sequenced data generated, when necessary, for extending the nucleotide sequence. Primer sequences are available on request.

Reagents for RT-PCR were obtained from TaKaRa (RNA PCR Kit [avian myeloblastosis virus] version 2.1; TaKaRa, Shiga, Japan), and RT-PCR was performed following the manufacturer's recommended protocol.

Templates for sequencing the SLC7A7 gene were obtained using the PCR method with LA-Taq DNA polymerase (TaKaRa, Shiga, Japan). PCR conditions were as follows: hot start for 3 minutes at $96^{\circ} \mathrm{C} ; 35$ cycles of denaturing $\left(96^{\circ} \mathrm{C}, 30\right.$ seconds), annealing $\left(58^{\circ} \mathrm{C}, 30\right.$ seconds), and extension $\left(72^{\circ} \mathrm{C}, 2\right.$ minutes).
The PCR products were purified from a 1\% agarose gel and sequenced in both directions using an automated DNA sequencer (model 310) using the ABI Prism BigDye Terminator Cycle Sequencing Ready Reaction kit (both, Perkin-Elmer, Courtaboeuf, France).

Study design for vascular endothelial function. Studies were performed in an isolated and temperature-controlled $\left(22-24^{\circ} \mathrm{C}\right)$ room while the study subjects were in the fasting state. Heart rate and blood pressure were monitored continuously during the period of the study.

The vasodilator responses in the brachial arteries were measured using an ultrasound technique as described by others $(14,25)$. Briefly, the diameter of the brachial artery was measured from B-mode ultrasound images with the use of a $7.5-\mathrm{MHz}$ linear-array transducer (model SSA-380A ultrasound system; Toshiba, Tokyo, Japan). The flow velocity of the brachial artery was measured by means of a pulsed Doppler signal at a $70^{\circ}$ angle to the vessel with the range gate $(1.5 \mathrm{~mm})$ in the center of the artery. The brachial artery was scanned in the antecubital fossa in a longitudinal fashion. When a satisfactory transducer position was located, the surface of the skin was marked, and the arm setting was optimized at the beginning of the study and was thereafter kept constant throughout the recording period.

The subjects lay quietly for 10 minutes before the start of the scan. Baseline measurements of the diameter and flow velocity in the brachial artery and blood sampling for the assay of L-arginine and $\mathrm{NO}$ derivatives $\left(\mathrm{NO}_{\mathrm{x}}\right)$ were performed 30 minutes after an intravenous infusion of saline at a rate of $6.7 \mathrm{ml} / \mathrm{min}$ for 30 minutes. After these baseline measurements, a blood pressure cuff was placed around the forearm and inflated to a pressure of $250-300 \mathrm{mmHg}$. After 4.5 minutes, the cuff was released. The diameter and flow velocity were continuously measured during cuff inflation and after cuff deflation. The flow-mediated dilator response was used as a measure of endothelium-dependent vasodilation (EDV). After a 15-minute rest, an intravenous L-arginine $(0.1 \mathrm{mg} / \mathrm{ml})$ infusion was started at a rate of $6.7 \mathrm{ml} / \mathrm{min}$ for 30 minutes. Thirty minutes after the end of the infusion, measurements of the diameter and the flow velocity in the brachial artery, and blood sampling were performed. EDV after L-arginine infusion was then measured. This protocol was performed three times at 1-week intervals on the patient, and once for each of the control A $(n=10)$. On a different day, the diameter was measured before and 4 minutes after the sublingual administration of nitroglycerin $(300 \mu \mathrm{g})$. The response to nitroglycerin was used as a measure of endotheliumindependent vasodilation (EIV). This protocol was performed five times at least 3-day intervals on the patient, and once for each of the control B $(n=10)$.

A blood sample from the patient's mother was also obtained and assayed for L-arginine and $\mathrm{NO}_{\mathrm{x}}$ three times while she was in the fasting state.

Data analysis. Images were recorded on a super-VHS videocassette recorder (SVO-9500MD; Sony Corp., Tokyo, Japan), and brachial arterial diameters were 
Table 1

Baseline characteristics of the patient and normal controls

\begin{tabular}{|c|c|c|c|c|c|}
\hline Characteristics & Control A $(n=10)$ & Control B $(n=10)$ & Patient & $P^{\mathrm{A}}$ & $P^{\mathrm{B}}$ \\
\hline Age $(y)$ & $35.3 \pm 4.2$ & $36.3 \pm 4.7$ & 37 & & \\
\hline Heart rate (beats/min) & $62.4 \pm 7.1$ & $67.4 \pm 7.8$ & $59.6 \pm 4.8$ & NS & NS \\
\hline Systolic blood pressure $(\mathrm{mmHg})$ & $119.8 \pm 7.5$ & $115.4 \pm 14.3$ & $116.2 \pm 1.9$ & NS & NS \\
\hline Diastolic blood pressure $(\mathrm{mmHg})$ & $67.0 \pm 5.4$ & $69.7 \pm 8.3$ & $75.2 \pm 1.9$ & NS & NS \\
\hline Body mass index $\left(\mathrm{kg} / \mathrm{m}^{2}\right)$ & $21.3 \pm 1.7$ & $22.8 \pm 1.8$ & 22.2 & & \\
\hline Cholesterol (mg/dl) & $172.0 \pm 26.4$ & $176.3 \pm 35.6$ & $190.0 \pm 12.5$ & NS & NS \\
\hline Fasting blood glucose $(\mathrm{mg} / \mathrm{dl})$ & $69.0 \pm 10.1$ & $72.0 \pm 6.0$ & $70.4 \pm 9.5$ & NS & NS \\
\hline
\end{tabular}

Values are expressed as mean \pm SD for control $A$, control $B$, and the patient. Patient was tested on five separate occasions. There are no significant differences between the controls (control A and control B) and the patient. ${ }^{A}$ Comparison of the control A and the patient by Welch's $t$ test. ${ }^{B}$ Comparison of the control $\mathrm{B}$ and the patient by Welch's $t$ test. Statistical significance is defined as $P<0.05$.

measured from the tape with ultrasonic calipers by two observers who were blinded as to the protocols of the study and the subject grouping. The arterial diameter was measured at a fixed distance from anatomic markers such as a bifurcation, facial plane, or vein observed in cross section by point-to-point measurements. Measurements were taken from the anterior to the posterior interface between media and adventitia ( $M$ line) at the end diastole, incident with the $\mathrm{R}$ wave on a continuously recorded electrocardiogram $(14,25)$. Diameters at four cardiac cycles were analyzed for each scan, and the measurements were averaged. Diameter measurements for reactive hyperemia were taken 45-90 seconds after cuff deflation. EDV was expressed as the percent change relative to that just prior to cuff inflation (taken as 100\%). EIV was expressed as the percent change relative to that just prior to nitroglycerin administration (taken as 100\%). EDV and EIV were calculated by each observer, and the average results of the two observations were recorded. Doppler flow signal and arterial diameter were simultaneously measured. Blood flow was calculated by multiplying the velocitytime integral of the Doppler flow signal by heart rate and the vessel cross-sectional area of the artery measured at that time. Reactive hyperemia was calculated as the maximum flow recorded in the first 15 seconds after cuff deflation and was divided by the flow during the first resting (baseline) scan $(14,25)$.

Determination of serum L-arginine and NO derivatives concentration. The concentration of serum L-arginine and $\mathrm{NO}$ derivatives was measured in control $\mathrm{A}$, in the patient, and in his mother.

Serum L-arginine concentrations were measured by means of an amino acid autoanalyzer (model L-8500 with column 2622SC; Hitachi, Tokyo, Japan).

Serum concentrations of NO derivatives nitrite and nitrate were assayed using a Sievers NO analyzer (Taiyo Toyo Sanso, Osaka, Japan) after reduction by vanadium trichloride (Wako Pure Chemicals Industries, Osaka, Japan) using the chemiluminescence method (26).

Treadmill test and coronary angiography. A treadmill test (TMT) was performed on the LPI patient, according to a modified Bruce protocol. TMT was also performed 30 minutes after an infusion of $200 \mathrm{ml}$ of $\mathrm{L}$-arginine $(0.1$ $\mathrm{mg} / \mathrm{ml}$ ) on another day. Coronary angiography (CAG) was performed as reported previously (27).

Positron emission tomography. Myocardial blood flow (MBF) was measured with ${ }^{13} \mathrm{~N}$-ammonia PET. The PET study was performed with a whole-body PET camera (SET-2400W [Headtome V]; Shimadzu Medico Co., Kyoto, Japan). The spatial resolution in the tomographic plane was $4 \mathrm{~mm}$ full-width half-maximum (FWHM) at the center, and the axial resolution was $5 \mathrm{~mm}$ FWHM.

The study protocol consisted of two study sessions, both of which were performed in the afternoon. MBF was measured at rest and after an adenosine triphosphate (ATP) infusion without or with an L-arginine infusion on different days in the LPI patient. On day 1 , beginning with intravenous ${ }^{13} \mathrm{~N}$-labeled ammonia administration $(740 \mathrm{MBq})$ in a cubital vein for $30 \mathrm{sec}-$ onds, dynamic emission images were acquired (12 frames of 10 seconds each and one frame of $600 \mathrm{sec}-$ onds). Fifty minutes were allowed for the decay of the radiotracer activity before the next image sequence was acquired. ATP was infused intravenously at a rate of $0.16 \mathrm{mg} / \mathrm{kg} / \mathrm{min}$ for 7 minutes using an infusion pump. Four minutes after the start of the ATP infusion, the administration of ${ }^{13} \mathrm{~N}$-ammonia $(740 \mathrm{MBq})$ and the image acquisition were started simultaneously. On another day, MBF was measured at rest, and ATP was infused 30 minutes after an infusion of $20 \mathrm{~g}$ of L-arginine. The administration of ${ }^{13} \mathrm{~N}$-ammonia and ATP, and image acquisition were identical to those performed on day $1(28,29)$.

Quantification of $M B F$. Regional MBF was calculated according to the two-compartment ${ }^{13} \mathrm{~N}$-ammonia tracer kinetic model (28). Twelve regions of interest (ROIs) were placed at the same intervals on a midlevel of shortaxis image. For each of the territories, three ROIs were averaged. The same anatomic landmark (anterior, lateral, inferior, septum) ensured the identical assignment of ROIs for the two-study conditions. A small ROI was centered in the left ventricular blood pool for a determination of the arterial input function. The ROIs were copied to the first 120 seconds of the serially acquired images for generation of the blood pool and myocardial time-activity curves. Tracer spillover was corrected by a least-square nonlinear regression analysis, and MBF was 


\section{Figure 1}

Schematic representation of the $S L C 7 A 7$ gene and mutations in the patient. (a) Structure of the SLC7A7 gene. The position of the CDNA is defined according to GenBank AJ130718. The coding sequence (CDS) is located in nucleotide numbers 294-1822. Exons (boxes); introns (bars). The numbers over the boxes are the first nucleotide of the exons. For example, " 1285 " indicates that nucleotide 1285 is the first nucleotide within exon 7. ATG indicates the initiation codon, and TAA depicts the stop codon. (b) In the maternal allele, an about 5.3-kbp Alu-mediated deletion from intron 2 to intron 4 was found, which results in skipping the entire exons 3 and 4 ( 271 bp). Left arm of the Alu repeat (open arrowheads); right arm of the Alu repeat (closed arrowheads). Broken lines and boxes indicate the deletion of the gene. (c) In the paternal allele, a point mutation occurred at the first nucleotide of intron 3 (IVS $3+1 \mathrm{G} \rightarrow \mathrm{A}$ ). This mutation destroys the GT donor splice site and leads to a splicing error, resulting in the skipping of the entire exon 3 (126 bp). The sequences of the splice donor and acceptor sites in the gene are also represented.

calculated with the assumption that the radioactivity from left ventricle to myocardium was influenced.

Coronary flow reserve (CFR) was defined as a ratio of myocardial blood flow during ATP infusion to the flow at rest.

MBF and CFR values for the LPI patient were compared with those of normal controls $(n=9)$.

Statistical analysis. The results are presented as means \pm SD. The statistical significance in comparisons between two measurements and among groups was determined with Welch's $t$ test. An ANOVA for the four groups was performed by the Kruskal-Wallis test, followed by Scheffé's test for multiple comparisons to enable pairwise testing for significant differences between groups. The statistical significance was defined as $P<0.05$.

\section{Results}

Mutations analysis of the SLC7A7 gene in the patient. The SLC7A7 gene is organized into ten exons with all the splice donor and acceptor sites conforming to the canonical GT/AG rule (30) (Figure 1a).

Two mutations were found in this patient. The first, inherited from his mother, is about a 5.3-kbp Alu-mediated deletion of genomic DNA from intron 2 to intron 4 , and cDNA revealed the deletion of all of exons 3 and 4 (271 bp) (Figure 1b). The sequences adjacent to the breakpoint were found to be part of two $A l u$ repeats that are located in intron 2 and 4, and which share an over-

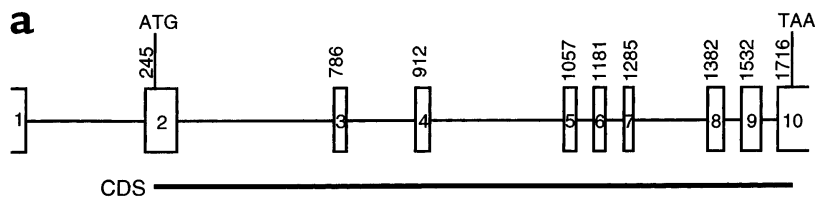

b

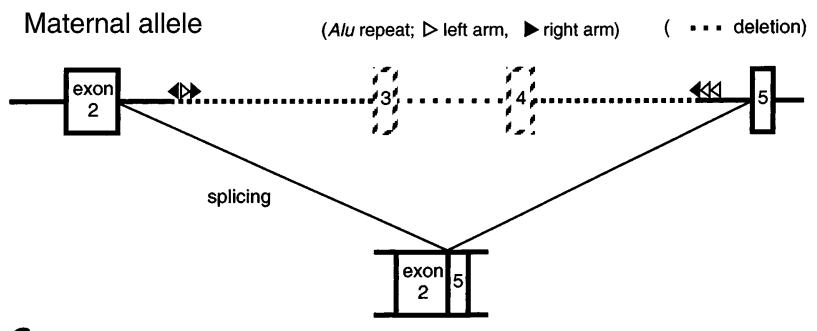

c

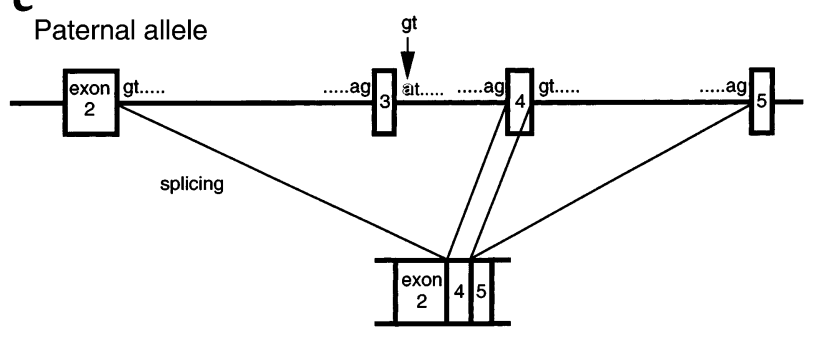

all homology of $66 \%$. The breakpoint sequence was a core 19-bp homologous sequence. The second mutation, assumed to be inherited from his father, is a $G \rightarrow A$ transition at the splice donor site of intron 3. This mutation has been reported by Sperandeo et al. in one Japanese LPI patient (30). The donor GT splice site was destroyed by this mutation, resulting in a splicing error at the former donor GT splice site of intron 2 and the deletion of all of exon 3 (126 bp) in the paternal cDNA (Figure 1c). Both of these two mutations generate a premature stop codon at codon 167 and eliminate the latter two thirds of the protein. Thus, our patient is a compound heterozygote for two mutations $(5.3-\mathrm{kbp}$ $A l u$-mediated deletion and IVS3 $+1 \mathrm{G} \rightarrow \mathrm{A}$ ). The maternal mutation is a novel one in the SLC7A7 gene.

Concentrations of L-arginine and NO derivatives. Serum Larginine concentration in the patient was markedly lower than that in control A $(21.1 \pm 4.3$ vs. $98.2 \pm 9.8$ $\mathrm{nmol} / \mathrm{ml} ; P<0.001$ ) (Figure 2a). The serum L-arginine concentration of the patient's mother was $92.5 \pm 6.1$ $\mathrm{nmol} / \mathrm{ml}$ (average of three determinations) that was within the normal range. The serum L-arginine concentrations of control $\mathrm{A}$ and the patient were both elevated

Table 2

Flow data at rest and at reactive hyperemia in the controls and in the patient before and after L-arginine infusion

\begin{tabular}{|c|c|c|c|c|c|c|c|}
\hline & Control A & Control A (Arg) & Patient & Patient $(\operatorname{Arg})^{\mathrm{B}}$ & $P$ value ${ }^{C}$ & $P$ value ${ }^{\mathrm{D}}$ & $P$ value $^{\mathrm{E}}$ \\
\hline Fow at rest $(\mathrm{m}$ & $288.0 \pm 31.3$ & $320.8 \pm 22.9$ & $245.8 \pm 8.0$ & $279.4 \pm 7.6$ & NS & NS & NS \\
\hline Baseline vessel size ( $\mathrm{mm})$ & $4.27 \pm 0.26$ & $4.43 \pm 0.30$ & $3.79 \pm 0.031$ & $4.05 \pm 0.012$ & NS & NS & NS \\
\hline Reactive hyperemia $(\mathrm{ml} / \mathrm{min})$ & $787.5 \pm 111.5$ & $874.9 \pm 98.7$ & $710.4 \pm 13.3$ & $830.0 \pm 26.9$ & NS & NS & NS \\
\hline Reactive hyperemia (\%) & $270.8 \pm 20.6$ & $281.4 \pm 28.5$ & $289.3 \pm 14.2$ & $297.0 \pm 3.3$ & NS & NS & NS \\
\hline
\end{tabular}

Values are expressed as mean $\pm \mathrm{SD}$. ${ }^{\mathrm{A} C}$ Control $\mathrm{A}$ after L-arginine infusion. ${ }^{\mathrm{B}}$ Patient after L-arginine infusion. ${ }^{\mathrm{C}} \mathrm{Comparison}$ of the control $\mathrm{A}$ and control $\mathrm{A}$ (Arg) infusion by Scheffé's test. DComparison of the control A and the patient by Scheffé's test. EComparison of the control A and the patient (Arg) by Scheffé's test. Statistical significance is defined as $P<0.05$. 

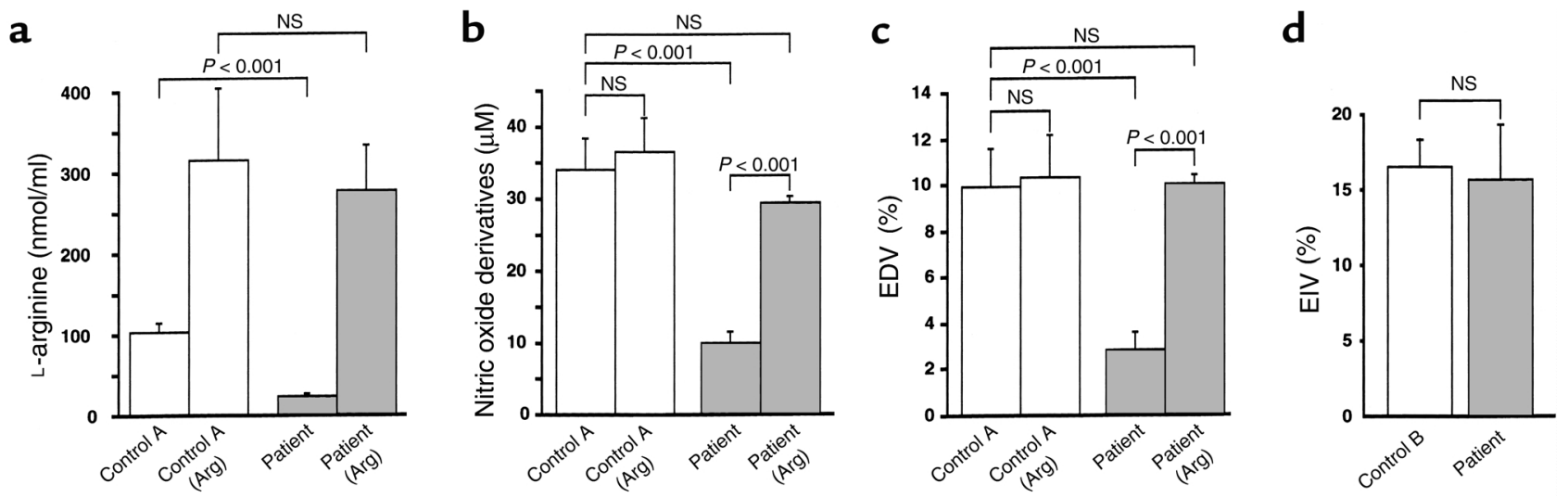

Figure 2

Comparison of L-arginine and NO derivative concentrations, and the vascular endothelial function between the normal controls and the patient. (a) Serum L-arginine concentration $(\mathrm{nmol} / \mathrm{ml})$ in normal controls $(n=10$; control A) and in the patient (three independent determinations) before (control A, patient) and after (control A [Arg], patient [Arg]) L-arginine infusion. Values represented are the mean \pm SD. A statistical analysis was determined using Welch's $t$ test. (b) Serum NO derivative concentrations $(\mu \mathrm{M})$ in normal controls $(n=10$; control A) and the patient (three independent determinations) before (control A, patient) and after (control A [Arg], patient [Arg]) an L-arginine infusion. Values shown are the mean \pm SD. A statistical analysis among the four groups was performed using the Kruskal-Wallis test $(P=0.001)$, followed by Scheffés test for multiple comparisons to allow pairwise testing for significant differences between the groups. (c) Percent increase in endothelium-dependent vasodilation (EDV) in normal controls $(n=10$; control $\mathrm{A})$ and the patient (three independent determinations) before (control $\mathrm{A}$, patient) and after (control A [Arg], patient [Arg]) an L-arginine infusion. Values represented are the mean \pm SD. A statistical analysis among the four groups was performed by the Kruskal-Wallis test $(P=0.049)$, followed by Scheffé's test for multiple comparisons to enable pairwise testing for significant differences between the groups. (d) Percent increase in EIV after sublingual nitroglycerin $(300 \mathrm{mg})$ in normal controls $(n=10$; control B) and in the patient (five independent determinations). Values represented are mean \pm SD. A statistical analysis was determined using the Welch's $t$ test.

30 minutes after an L-arginine infusion $(298.1 \pm 82.3$ vs. $260.3 \pm 54.0 \mathrm{nmol} / \mathrm{ml} ; P=\mathrm{NS}$ ) (Figure 2a).

The fasting serum $\mathrm{NO}_{\mathrm{x}}$ level in the patient with LPI was markedly lower than that of the control A $(9.93 \pm 1.56 \mu \mathrm{M} ; P<0.0001)$. The serum $\mathrm{NO}_{\mathrm{x}}$ level in the patient was significantly elevated after an intravenous administration of $\mathrm{L}$-arginine $(29.37 \pm 0.85 \mu \mathrm{M})$. No significant difference in the serum $\mathrm{NO}_{\mathrm{x}}$ level in control A was detected before $(34.0 \pm 4.47 \mu \mathrm{M})$ and after $(36.5 \pm 4.89 \mu \mathrm{M})$ an L-arginine infusion (Figure $2 \mathrm{~b}$ ).

The serum $\mathrm{NO}_{\mathrm{x}}$ level in the patient's mother was $32.1 \pm 2.7 \mu \mathrm{M}$ (average of three determinations) which was not statistically different from those in the normal controls.

Vascular endothelial function. No significant difference in the flow at rest and after reactive hyperemia was evident between control A and the patient before and after an L-arginine infusion (Table 2).

The EDV in the patient before L-arginine infusion was markedly impaired compared with that for the control A $(2.80 \% \pm 0.76 \%$ vs. $9.95 \% \pm 1.65 \% ; P<0.0001)$. After the intravenous infusion of $\mathrm{L}$-arginine, the EDV in the patient was elevated to $10.01 \% \pm 0.30 \%$. No enhancement of EDV with L-arginine in control A was detected $(10.31 \% \pm 1.83 \%)$ (Figure $2 \mathrm{c}$ ).

The EIV was not significantly different between the patient and control B $(15.74 \% \pm 2.87 \%$ vs. $16.51 \% \pm 2.46 \%$; $P=\mathrm{NS}$ ) (Figure 2d). The interobserver variability in the measurements of arterial diameter in this study was $0.04 \pm 0.01 \mathrm{~mm}(n=41)$.

TMT and CAG. An electrocardiograph (ECG) of the patient at rest was normal. The TMT without L-arginine infusion was discontinued because of chest pain. The TMT revealed a $0.15 \mathrm{mV}$ horizontal ST depression in leads II, III, aVf, V4, V5, and V6 at 80 microseconds after the J point. The onset of depression of $0.1 \mathrm{mV}$ was at 7.5 minutes of exercise, persisting until 3 minutes in the recovery period. The peak heart rate was 150 beats per minute, and the peak blood pressure was $157 / 74$ $\mathrm{mmHg}$, giving a double product of 23,550 . After the L-arginine infusion, the patient reached the target heart rate without chest pain, and no ST depression was detected. The peak heart rate was 178 beats per minute, and the peak blood pressure was $169 / 87 \mathrm{mmHg}$, giving a double product of 30,082 .
Table 3

Myocardial blood flow and coronary flow reserve in the LPI patient and normal controls

\begin{tabular}{|c|c|c|c|c|c|c|c|}
\hline & & ant & lat & $\inf$ & sep & mean value ${ }^{A}$ & $\begin{array}{c}\text { mean value of } \\
\text { controls }(n=9)\end{array}$ \\
\hline $\operatorname{Arg}(-)^{B}$ & $\begin{array}{l}\text { at rest }(\mathrm{ml} / \mathrm{g} / \mathrm{min}) \\
\text { ATP }(\mathrm{ml} / \mathrm{g} / \mathrm{min}) \\
\text { CFR }\end{array}$ & $\begin{array}{l}0.84 \\
1.62 \\
1.92\end{array}$ & $\begin{array}{l}0.79 \\
1.78 \\
2.25\end{array}$ & $\begin{array}{l}0.96 \\
1.84 \\
1.93\end{array}$ & $\begin{array}{l}0.59 \\
1.35 \\
2.29\end{array}$ & $\begin{array}{l}0.80 \\
1.65 \\
2.10\end{array}$ & $\begin{array}{l}0.57 \pm 0.08 \\
1.98 \pm 0.46 \\
3.56 \pm 0.65\end{array}$ \\
\hline $\operatorname{Arg}(+)^{B}$ & $\begin{array}{l}\text { at rest }(\mathrm{ml} / \mathrm{g} / \mathrm{min}) \\
\text { ATP }(\mathrm{ml} / \mathrm{g} / \mathrm{min}) \\
\text { CFR }\end{array}$ & $\begin{array}{l}0.61 \\
2.58 \\
4.23\end{array}$ & $\begin{array}{l}0.62 \\
2.85 \\
4.60\end{array}$ & $\begin{array}{l}0.75 \\
2.81 \\
3.75\end{array}$ & $\begin{array}{l}0.52 \\
2.46 \\
4.73\end{array}$ & $\begin{array}{l}0.63 \\
2.68 \\
4.33\end{array}$ & \\
\hline
\end{tabular}

Values are expressed as mean \pm SD. ${ }^{A}$ Mean value of anterior, lateral, inferior wall, and septum. ${ }^{B}$ Without or with L-arginine infusion. Ant, anterior; lat, lateral; inf, inferior wall; and sep, septum. 

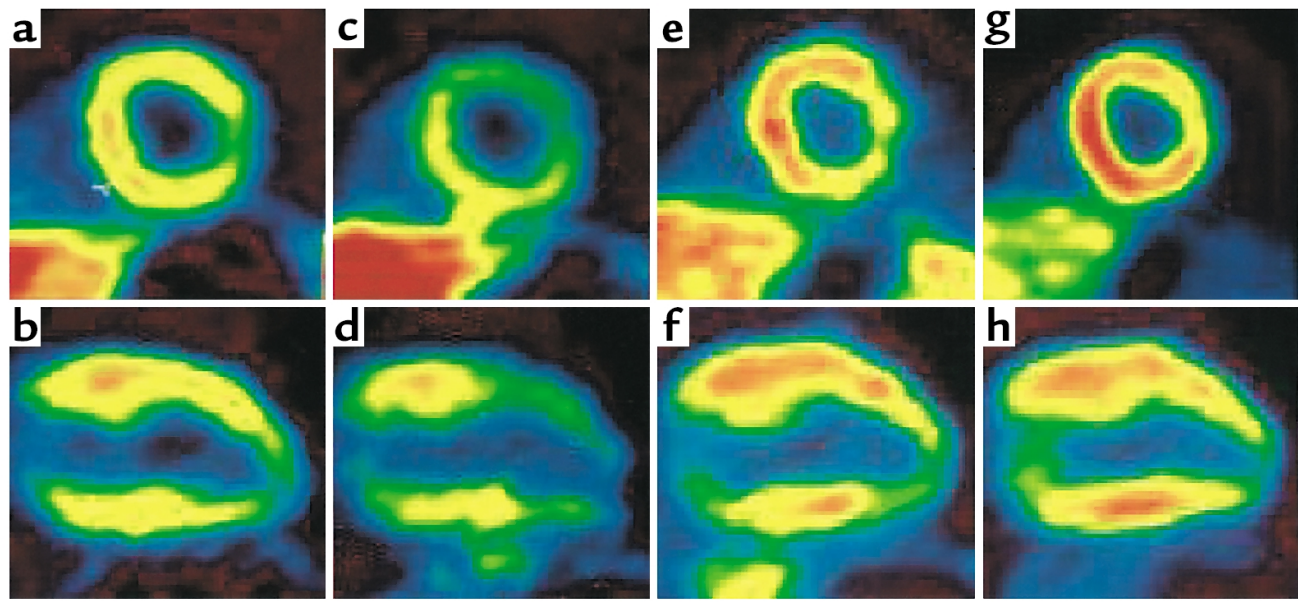

\section{Figure 3}

PET of heart in the patient before and after L-arginine infusion. Coronary artery function was investigated in the patient, using PET of the heart with ${ }^{13} \mathrm{~N}$-ammonia at baseline ( $\mathbf{a}$ and $\mathbf{b}$ ) and after adenosine triphosphate (ATP) infusion ( $\mathbf{c}$ and $\left.\mathbf{d}\right)$. On another day, we also investigated the coronary artery function in the patient, using PET of the heart with ${ }^{13} \mathrm{~N}$-ammonia 30 minutes after a 200 -ml L-arginine $(0.1 \mathrm{mg} / \mathrm{ml})$ infusion at the baseline (e and $\mathbf{f})$ and after an ATP infusion ( $\mathbf{g}$ and $\mathbf{h})$. The short axis images (a, $\mathbf{c}, \mathbf{e}$, and $\mathbf{g})$ and the vertical long axis images $(\mathbf{b}, \mathbf{d}, \mathbf{f}$, and $\mathbf{h})$ are shown.

His CAG revealed a normal coronary artery with no atherosclerotic irregularities.

PET study. The MBF and CFR values for the patient are listed in Table 3 . At rest, no reduction was observed in the average MBF value for the patient $(0.80$ $\mathrm{ml} / \mathrm{g} / \mathrm{min}$ and $0.63 \mathrm{ml} / \mathrm{g} / \mathrm{min})$. However, the average MBF value at ATP infusion of the patient was lower than that of the control $(1.65 \mathrm{ml} / \mathrm{g} / \mathrm{min}$ vs. $1.98 \pm 0.46$ $\mathrm{ml} / \mathrm{g} / \mathrm{min}$ ). The MBF value at ATP infusion of the patient was reduced especially in the anterior wall. The average CFR value was markedly lower in the patient than that in the normal control (2.10 vs. $3.56 \pm 0.65)$. The MBF and CFR values were both significantly improved after L-arginine infusion (2.68 and 4.33, respectively) (Table 3; Figure 3).

\section{Discussion}

The present study showed that an L-arginine deficiency causes vascular endothelial dysfunction via a decrease in NO production in this LPI patient. NO plays an important role in vascular endothelial function. Because L-arginine is the only substrate for the NOScatalyzed reaction (18), the availability of this substrate is, therefore, critical in regulating NO production. The rate of NO formation is dependent on the concentration of L-arginine (31). A study of vascular endothelial function in an L-arginine-deficient state is important in terms of clarifying the role of L-arginine in vascular function. However, no studies of endothelial function in an L-arginine deficiency in vivo are currently available. Because L-arginine is, nutritionally speaking, a nonessential amino acid for adult mammals, including humans (19), L-arginine-deficient animals are not readily available for in vivo studies. Only two investigations have reported the effect of an L-arginine deficiency on NO synthesis in vivo. One involved the use of an animal model using rats, in conjunction with a massive resection of the small intestine (30), the other is the case of a human subject with LPI that we reported previously (20). Palmer et al. reported that bovine aortic endothelial cells, when cultured in a medium deficient in L-arginine, released little NO (32). In the case of LPI patients, serum L-arginine concentration is very low, although the enzymes of the urea cycle have normal activities (22). To elucidate the relationship between an L-arginine deficiency and vascular endothelial function, NO levels and vascular endothelial function were examined for an LPI patient with an L-arginine deficiency.

LPI is a rare autosomal recessive disease caused by an abnormality in the dibasic amino acid transporter $\left(y^{+} \mathrm{L}\right.$ amino acid transporter $1 ; y^{+}$LAT1) at the basolateral (antiluminal) membrane of the epithelial cell in small intestine and renal tubules (22). The abnormality of the $\mathrm{y}^{+}$LAT1 leads to an impairment in intestinal absorption and a renal loss of dibasic amino acids (DAA; L-arginine, L-ornithine, L-lysine), resulting in a deficiency of DAA in LPI patients. Recently, the SLC7A7 cDNA that encodes for the $y^{+} L A T 1$ has been cloned, and mutations in this gene have been reported in LPI patients $(23,24,30,33)$. The findings herein show that the patient is a compound heterozygote for the two SLC7A7 gene mutations. The mutation in the maternal allele is a large Alu-mediated deletion in the SLC7A7 gene. This represents a novel mutation. The paternal mutation is a splicing mutation in the gene (IVS3+1 G $\rightarrow \mathrm{A}$ ). This is the same mutation that Sperandeo et al. reported in another Japanese LPI patient (30). Mykkanen et al. reported that some of the LPI mutations, including missense mutations $(1287 \mathrm{~T} \rightarrow \mathrm{G})$ and short deletion (1291del CTTT, 1548delC), showed no enhancing effect of amino acid transport function with $4 \mathrm{~F} 2 \mathrm{hc}$ (the surface antigen 4F2 heavy chain) (33). Both mutations in our patient 
results in the loss of the latter two thirds of the $y^{+}$LAT1, which includes these mutation sites. Although we did not measure the $y^{+} \mathrm{L}$ transport activity, it is likely that both mutations also would be expected to lead to no or very low $\mathrm{y}^{+} \mathrm{LAT} 1$ activity. The reduced serum concentration and increased urinary excretion of $\mathrm{L}$-arginine in this patient confirmed that he has a chronic deficiency of L-arginine, as we reported previously (20).

$\mathrm{NO}_{\mathrm{x}}$ levels were determined by the chemiluminescence method. This is the method by which Palmer et al. measured NO from endothelial cells for the first time (3). The mean $\mathrm{NO}_{\mathrm{x}}$ concentration of the normal control in this study was $34.0 \mu \mathrm{M}$. This is in nearly the same range as in other previous reports $(26,34)$. The $\mathrm{NO}_{x}$ concentration in our patient was reduced to $32 \%$ of that in the normal controls. The decline in plasma $\mathrm{NO}_{\mathrm{x}}$ levels is as significant as that of $N^{\mathrm{G}}$-monomethyl-L-arginine (L-NMMA; $1 \mathrm{mg} / \mathrm{kg} / \mathrm{min}$ ), which, when infused into a human, reduced plasma $\mathrm{NO}_{x}$ levels to $35 \%$ (35). The same effect could be the result of an impaired denitrosylation of derivatized end products. However, the finding that NO derivatives were elevated to a normal level after $\mathrm{L}$-arginine infusion suggests that the decrease in $\mathrm{NO}_{x}$ levels in the patient was caused by the low serum L-arginine.

In the patient's mother, a heterozygote for the SLC7A7 gene (5.3-kbp Alu-mediated deletion), the concentrations of L-arginine in the blood and urine were normal, as was the $\mathrm{NO}_{\mathrm{x}}$ level in the blood. This indicates that the apparent L-arginine metabolism is not altered in a heterozygote for the mutation observed in the patient's mother.

The reason for why the blood pressure of the patient is normal contrary to such a low concentration of NO derivatives remains unknown. Some presently unidentified system might compensate for the blood pressure in the case of a patient in a state of chronic NO deficiency. In LPI patients, not only L-arginine but also L-lysine is deficient. L-Lysine is a competitive antagonist of L-arginine uptake, and Simmons et al. reported that L-lysine suppressed NO production (36). A lysine deficiency causes poor growth and prominent osteoporosis, and L-lysine administration is tolerated well by patients with LPI (37). However, supplementation of only L-lysine in LPI patients would cause a more reduced NO production.

We previously reported a decrease in the platelet count and an increase in the plasma levels of the thrombinantithrombin III complex (TAT) and fibrin degradation products (FDPs) in this patient (20). The intravenous administration of $\mathrm{L}$-arginine or transdermal nitroglycerin administration increased the platelet count and reduced plasma TAT and FDP levels. The reduced NO concentration in the LPI patient increased after L-arginine administration. These results indicate that intravascular coagulation is exacerbated by an L-arginine deficiency in the LPI patient via a reduced NO production. This is the first report that clarifies the pathophysiology caused by a decreased NO production in an LPI patient.

In the current study, vascular endothelial function was investigated using an ultrasound technique. Arte- rial dilatation in response to flow increase has been shown to be dependent on endothelial function $(7,38)$. Endothelium-dependent vascular dilatation is largely due to the release of $\mathrm{NO}$, and flow-dependent dilatation of the human peripheral conduit artery can be used as a test for the L-arginine/NO pathway in clinical studies $(7,9)$. The increase in brachial arterial diameter in normal controls $(9.95 \% \pm 1.65 \%)$ in this study is nearly the same as that reported by others $(8.2 \%-11 \%)(14$, $25,39)$. The administration of L-arginine in healthy controls did not cause an enhancement in EDV as reported in a previous study (40).

The increase in brachial arterial diameter in the patient was reduced to $28.1 \%$ of that in the controls. This is as severe as that observed for patients with coronary artery disease (28\%) (41) and is more severe than that reported for chronic smokers (52\%) (14). An abnormality in endothelium-dependent arterial reactivity has been reported in hypertension (10), hypercholesterolemia (11-13), chronic smoking (14), diabetes mellitus (15), and coronary artery disease $(16,17)$, all of which were excluded in the subjects participating in the present study. Because the response to nitroglycerin, a direct relaxant of smooth muscle, was preserved in the patient, the data herein suggest that the reduced L-arginine concentration in the LPI patient was the cause of the vascular endothelial dysfunction. Consistent with this hypothesis, the supplementation of L-arginine normalized both NO concentrations and vascular endothelial function in the patient. To the best of our knowledge, this is the first report that clearly shows that a deficiency of L-arginine causes vascular endothelial dysfunction via a decrease in NO production in humans.

Endothelial NOS (eNOS) gene polymorphism is associated with plasma NO metabolite levels in humans (42), and a Japanese group reported that mutations in the eNOS gene are associated with coronary spasms (43, 44). L-NMMA decreases NO production in humans in vivo (45) and reduces the luminal diameter of coronary arteries in healthy humans in a dose-dependent manner (46). Coronary artery function was tested using a TMT and a PET of heart in the LPI patient. Both the TMT and PET revealed ischemic changes, which were improved by an L-arginine infusion. The patient's chest pain was also improved after L-arginine infusion. No coronary artery stenosis or atherosclerotic changes were observed in coronary arteriography. This indicates that the ischemic symptoms and positive TMT and PET studies observed in this individual were due to alterations in vascular reactivity.

L-Arginine improves endothelial function in cardiovascular diseases such as coronary heart disease $(16,17)$ or in risk factors such as hypercholesterolemia (11-13) or diabetes mellitus (15). A lack of L-arginine has been suggested as a possible cause in patients with syndrome $\mathrm{X}$ (patients with angina pectoris and a normal coronary arteriogram), and Egashira et al. reported that L-arginine supplementation improved endothelium-dependent coronary vasodilation in patients with syndrome $X$ 
(47). Lerman et al. proposed the use of L-arginine as a therapeutic option for patients with coronary endothelial dysfunction (48). Our results provide support for the benefit of L-arginine in terms of improving vascular endothelial function. In conclusion, we were able to show that an L-arginine deficiency caused vascular endothelial dysfunction via a decrease in $\mathrm{NO}$ production in an LPI patient.

\section{Acknowledgments}

We thank all the volunteers who participated in this study, and the staff of the Department of Internal Medicine and Molecular Science for their help. We also thank Shinji Hasegawa (Division of Tracer Kinetics, Biomedical Research Center, Osaka University Graduate School of Medicine) for advising us in the PET study.

1. Furchgott, R.F., and Zawadzki,J.V. 1980. The obligatory role of endothelial cells in the relaxation of arterial smooth muscle by acetylcholine. Nature. 228:373-376.

2. Ignarro, L.J., Buga, G.M., Wood, K.S., Byrns, R.E., and Chaudhuri, G. 1987. Endothelium-derived relaxing factor produced and released from artery and vein is nitric oxide. Proc. Natl. Acad. Sci. USA. 84:9265-9269.

3. Palmer, R.M.J., Ferrige, A.G., and Moncada, S. 1987. Nitric oxide release accounts for the biological activity of endothelium-derived relaxing factor. Nature. 327:524-526.

4. Dalziel, H.H., Thornbury, K.D., Ward, S.M., and Sanders, K.M. 1991. Involvement of nitric oxide synthetic pathway in inhibitory junction potentials in canine proximal colon. Am. J. Physiol. 260:G789-G792.

5. Stuehr, D.J., and Nathan, C.F. 1989. Nitric oxide: a macrophage product responsible for cytostasis and respiratory inhibition in tumor target cells. J. Exp. Med. 169:1543-1545.

6. de Graaf, J.C., et al. 1992. Nitric oxide functions as an inhibitor of platelet adhesion under flow conditions. Circulation. 85:2284-2290.

7. Rubanyi, G.M., Romero, J.C., and Vanhoutte, P.M. 1986. Flow-induced release of endothelium-derived relaxing factor. Am. J. Physiol. 250:H1145-H1149.

8. Quyyumi, A.A., et al. 1997. Nitric oxide activity in the atherosclerotic human coronary circulation. J. Am. Coll. Cardiol. 29:308-317.

9. Jonannides, R., et al. 1995. Nitric oxide is responsible for flow-dependent dilatation of human peripheral conduit arteries in vivo. Circulation. 91:1314-1319.

10. Imaizumi, T., et al. 1992. Effects of L-arginine on forearm vessels and responses to acetylcholine. Hypertension. 20:511-517.

11. Clarkson, P., et al. 1996. Oral L-arginine improves endothelium-dependent dilation in hypercholesterolemic young adults. J. Clin. Invest. 97:1989-1994.

12. Drexler, H., Zeiher, A.M., Meinzer, K., and Just, H. 1991. Correction of endothelial dysfunction in coronary microcirculation of hypercholesterolaemic patients by L-arginine. Lancet. 338:1546-1550.

13. Creager, M.A., et al. 1992. L-Arginine improves endothelium-dependent vasodilation in hypercholesterolemic humans. J. Clin. Invest. 90:1248-1253.

14. Motoyama, T., et al. 1997. Endothelium-dependent vasodilation in the brachial artery is impaired in smokers: effect of vitamin C. Am. J. Physiol. 273:H1644-H1650.

15. Smulders, R.A., et al. 1994. Plasma endothelin levels and vascular effects of intravenous L-arginine infusion in subjects with uncomplicated insulindependent diabetes mellitus. Clin. Sci. (Lond). 87:37-43.

16. Adams, M.R., et al. 1997. Oral L-arginine improves endothelium-dependent dilatation and reduces monocyte adhesion to endothelial cells in young men with coronary artery disease. Atherosclerosis. 129:261-269.

17. Kuo, L., Davis, M.J., Cannon, M.S., and Chilian, W.M. 1992. Pathophysiological consequences of atherosclerosis extend into the coronary microcirculation. Restoration of endothelium-dependent responses by L-arginine. Circ. Res. 70:465-476.

18. Morris, S.M., Jr., and Billiar, T.R. 1994. New insights into the regulation of inducible nitric oxide synthesis. Am. J. Physiol. 266:E829-E839.

19. Irwin, M.I., and Hegsted, D.M. 1971. A conspectus of research on amino acid requirements of man. J. Nutr. 101:539-566.

20. Kayanoki, Y., et al. 1999. Reduced nitric oxide production by L-arginine deficiency in lysinuric protein intolerance exacerbates intravascular coagulation. Metabolism. 48:1136-1140.

21. Ribeiro, M.O., Antunes, E., DeNucci, G., Lovisolo, S.M., and Zatz, R. 1992. Chronic inhibition of nitric oxide synthesis: a new model of arterial hypertension. Hypertension. 20:298-303.
22. Simell, O. 1995. Lysinuric protein intolerance and other cationic aminoacidurias. In The metabolic and molecular bases of inherited disease. C.H. Scriver, A.L. Beaudet, W.S. Sly, and D. Valle, editors. McGraw-Hill. New York, New York, USA. 3603-3627.

23. Torrents, D., et al. 1999. Identification of SLC7A7, encoding $y^{+} L A T-1$, as the lysinuric protein intolerance gene. Nat. Genet. 21:293-296.

24. Borsani, G., et al. 1999. SLC7A7, encoding a putative permease-related protein, is mutated in patients with lysinuric protein intolerance. Nat. Genet. 21:294-301.

25. Celermajer, D.S., et al. 1992. Non-invasive detection of endothelial dysfunction in children and adults at risk of atherosclerosis. Lancet. 340:1111-1115.

26. Braman, R.S., and Hendrix, S.A. 1989. Nanogram nitrite and nitrate determination in environmental and biological materials by vanadium(III) reduction with chemiluminescence detection. Anal. Chem. 61:2715-2718.

27. Maeda, N., et al. 1997. Acetylcholine-induced coronary microvascular vasospasm in a patient with angina pectoris and normal coronary angiogram: a case report. Angiology. 48:995-999.

28. Bottcher, M., Botker, H.E., Sonne, H., Nielsen, T.T., and Czernin, J. 1999. Endothelium-dependent and -independent perfusion reserve and the effect of L-arginine on myocardial perfusion in patients with syndrome X. Circulation. 99:1795-1801.

29. Takeishi, Y., et al. 1998. Myocardial tomography with technetium-99mtetrofosmin during intravenous infusion of adenosine triphosphate. J. Nucl. Med. 39:582-586.

30. Sperandeo, M.P., et al. 2000. Structure of the SLC7A7 gene and mutational analysis of patients affected by lysinuric protein intolerance. Am.J. Hum. Genet. 66:92-99.

31. Kilbourn, RG., and Belloni, P. 1990. Endothelial cell production of nitrogen oxides in response to interferon $\gamma$ in combination with tumor necrosis factor, interleukin-1, of endotoxin. J. Natl. Cancer Inst. 82:772-776.

32. Palmer, R.M.J., Ress, D.D., Ashton, D.S., and Moncada, S. 1988. L-Arginine is the physiological precursor for the formation of nitric oxide in endothelium-dependent relaxation. Biochem. Biophys. Res. Commun. 153:1251-1256.

33. Mykkanen, J., et al. 2000. Functional analysis of novel mutations in $y+$ LAT1 amino acid transporter gene causing lysinuric protein intolerance (LPI). Hum. Mol. Genet. 9:431-438.

34. Guevara, I., et al. 1998. Determination of nitrite/nitrate in human biological material by the simple Griess reaction. Clin. Chim. Acta. 274:177-188.

35. Stamler, J.S., Loh, E., Roddy, M.A., Currie, K.E., and Creager, M.A. 1994. Nitric oxide regulates basal systemic and pulmonary vascular resistance in healthy humans. Circulation. 89:2035-2040.

36. Simmons, W.W., Closs, E.I., Cunningham, J.M., Smith, T.W., and Kelly, R.A. 1996. Cytokines and insulin induce cationic amino acid transporter (CAT) expression in cardiac myocytes. J. Biol. Chem. 271:11694-11702.

37. Lukkarinen, M., Nanto-Salonen, K., Pulkki, K., Mattila, K., and Simell, O. 2000. Effect of lysine infusion on urea cycle in lysinuric protein intolerance. Metabolism. 49:821-825.

38. Pohl, U., Holz, J., Busse, R., and Bassenge, E. 1986. Crucial role of endothelium in the vasodilator response to increased flow in vivo. Hypertension. 8:37-44.

39. Celermajer, D.S., et al. 1996. Passive smoking and impaired endotheliumdependent arterial dilatation in healthy young adults. N. Engl. J. Med. 334:150-154.

40. Adams, M.R., Forsyth, C.J., Jessup, W., Robinson, J., and Celermajer, D.S. 1995. Oral L-arginine inhibits platelet aggregation but does not enhance endothelium-dependent dilation in healthy young men. J. Am. Coll. Cardiol. 26:1054-1061.

41. Anderson, T.J., et al. 1995. Close relation of endothelial function in human coronary and peripheral circulations. J. Am. Coll. Cardiol. 26:1235-1241.

42. Tsukada, T., et al. 1998. Evidence of association of the ecNOS gene polymorphism with plasma NO metabolite levels in humans. Biochem. Biophys. Res. Commun. 245:190-193.

43. Nakayama, M., et al. 1999 . $\mathrm{T}^{-786} \rightarrow \mathrm{C}$ mutation in the $5^{\prime}$-flanking region of the endothelial nitric oxide synthase gene is associated with coronary spasm. Circulation. 99:2864-2870.

44. Yoshimura, M., et al. 1998. A missense Glu298Asp variant in the endothelial nitric oxide synthase gene is associated with coronary spasm in the Japanese. Hum. Genet. 103:65-69.

45. Vallance, P., et al. 1995. Direct measurement of nitric oxide in human beings. Lancet. 345:153-154.

46. Kugiyama, K., et al. 1996. Nitric oxide activity is deficient in spasm arteries of patients with coronary spastic angina. Circulation. 94:266-272.

47. Egashira, K., Hirooka, Y., Kuga, T., Mohri, M., and Takeshita, A. 1996. Effects of L-arginine supplementation on endothelium-dependent coronary vasodilation in patients with angina pectoris and normal coronary arteriograms. Circulation. 94:130-134.

48. Lerman, A., Burnett, J.C., Jr., Higano, S.T., Mckinley, L.J., and Holmes, D.R., Jr. 1998. Long-term L-arginine supplementation improves small-vessel coronary endothelial function in humans. Circulation. 97:2123-2128. 